Tôhoku Math. Journ.

39 (1987), 589-596.

\title{
ESTIMATES OF THE AREA INTEGRALS BY THE NON-TANGENTIAL MAXIMAL FUNCTIONS
}

\author{
MAKOTO KANEKO
}

(Received October 1, 1986)

Introduction. In this paper, we give a method to estimate the area integrals by the non-tangential maximal functions.

For a harmonic function $u$ on the upper half-space $\boldsymbol{R}_{+}^{n+1}=\boldsymbol{R}^{n} \times(0, \infty)$, the area integrals $A_{\alpha} u$ and the non-tangential maximal functions $N_{\alpha} u$ are defined by

$$
\begin{aligned}
& \left(A_{\alpha} u\right)(x)=\left\{\int_{\Gamma_{\alpha}(x)}|(\nabla u)(y, s)|^{2} s^{1-n} d y d s\right\}^{1 / 2} \text { and } \\
& \left(N_{\alpha} u\right)(x)=\sup \left\{|u(y, s)| ;(y, s) \in \Gamma_{\alpha}(x)\right\},
\end{aligned}
$$

respectively, for $\alpha>0$ and $x \in \boldsymbol{R}^{n}$. In the above formulas, $|\nabla u|^{2}=$ $\sum\left|\partial u / \partial x_{j}\right|^{2}+|\partial u / \partial t|^{2}$ with the summation taken over $j=1, \cdots, n$, and $\Gamma_{\alpha}(x)=\left\{(y, s) \in \boldsymbol{R}_{+}^{n+1} ;|x-y|<\alpha s\right\}$, where $|x|$ is the Euclidean norm of $x \in \boldsymbol{R}^{n}$. We denote the points of $\boldsymbol{R}_{+}^{n+1}$ by the ordered pairs $(x, t)$, where $x \in \boldsymbol{R}^{n}$ and $t$ is a positive real.

The norm equivalence between $A_{\alpha} u$ and $N_{\alpha} u$ was obtained by Burkholder-Gundy [1] and Fefferman-Stein [4].

If $u$ is harmonic in $R_{+}^{n+1}$, then $|\nabla u|^{2}=\Delta|u|^{2} / 2$, where $\Delta$ denotes the Laplacian on $\boldsymbol{R}_{+}^{n+1}, \sum \partial^{2} / \partial x_{j}^{2}+\partial^{2} / \partial t^{2}$ with the summation taken over $j=$ $1, \cdots, n$. Furthermore, $|u|^{2}$ is subharmonic in $\boldsymbol{R}_{+}^{n+1}$.

McConnell [7] introduced certain functions $S_{\alpha} v$ on $\boldsymbol{R}^{n}$ for a subharmonic function $v$ on $\boldsymbol{R}_{+}^{n+1}$. It can be written as

$$
\left(S_{\alpha} v\right)(x)=\int_{\Gamma_{\alpha}(x)}(\Delta v)(y, s) s^{1-n} d y d s,
$$

if $v \in C^{2}\left(\boldsymbol{R}_{+}^{n+1}\right)$. He proved $\left\|S_{\alpha} v\right\|_{p} \leqq C\left\|N_{\alpha} v\right\|_{p}$ for a limited range of $p$, where $\|\cdot\|_{p}$ denotes the $L^{p}\left(\boldsymbol{R}^{n}\right)$-norm and $C$ is a constant depending only on $n, \alpha, p$. Recently, Uchiyama [9] obtained the same result for all $0<p<\infty$.

We shall give another proof for this latter result and show that our method is applicable to more general area integrals including the ones

This work was partly supported by the Grant-in-Aid for Scientific Research, the Ministry of Education, Science and Culture, Japan. 
induced from temperatures, which were studied in [2].

The author thanks Professor A. Uchiyama and the referee for their useful advice.

1. Definitions and the main theorem. Let $A=\left(a_{j k}\right)$ be an $(n+1)$ dimensional complex square matrix and $b_{1}, \cdots, b_{n+1}$ be complex numbers. We define the differential operator $L$ by

$$
L=\sum_{j, k=1}^{n+1} \alpha_{j k} \frac{\partial^{2}}{\partial x_{j} \partial x_{k}}+\sum_{j=1}^{n+1} b_{j} t^{-1} \frac{\partial}{\partial x_{j}},
$$

where, and hereafter, $x_{n+1}$ denotes the $(n+1)$-st variable $t$.

As the following examples show, it may be natural to consider the following area integrals. To a locally integrable function $v$ on $\boldsymbol{R}_{+}^{n+1}$, we apply the operator $L$ in the sense of distributions. We consider the case where $L v$ is a positive Borel measure $\mu_{L v}$ on $\boldsymbol{R}_{+}^{n+1}$. For such $v$, we define the area integrals $S_{\alpha} v$ by

$$
\left(S_{\alpha} v\right)(x)=\int_{\Gamma_{\alpha}(x)} s^{1-n} d \mu_{L v}(y, s) .
$$

If $A$ is non-negative and $b_{1}, \cdots, b_{n+1}$ are real, and if $u \in C^{2}\left(\boldsymbol{R}_{+}^{n+1}\right)$ is a solution of $L u=0$, then

$$
L|u|^{2}=([A+\bar{A}] \partial u / \partial x, \partial u / \partial x) \geqq 0,
$$

where $\bar{A}=\left(\bar{a}_{j_{k}}\right)$ and $\bar{a}_{j_{k}}$ is the complex conjugate of $a_{j k}, \partial u / \partial x$ denotes the column vector ${ }^{t}\left(\partial u / \partial x_{1}, \cdots, \partial u / \partial x_{n+1}\right)$ and $(\cdot, \cdot)$ is the usual inner product in $\boldsymbol{C}^{n+1}$.

EXAMPLE 1. If $u$ is harmonic in $R_{+}^{n+1}$ and $L=\Delta$, then $L u=0$, $L|u|^{2}=2|\nabla u|^{2}$ and $S_{\alpha}|u|^{2}=2\left(A_{\alpha} u\right)^{2}$.

EXAMPLE 2. When $u_{0}$ is a temperature on $\boldsymbol{R}_{+}^{n+1}$, that is to say, $u_{0}$ is infinitely differentiable and satisfies $\partial u_{0}(x, t) / \partial t=\Delta_{x} u_{0}(x, t)=$ $\sum_{j=1}^{n} \partial^{2} u_{0}(x, t) / \partial x_{j}^{2}$ in $\boldsymbol{R}_{+}^{n+1}$, by setting $u(x, t)=u_{0}\left(x, t^{2} / 4 \pi\right)$ an dtaking $L=$ $\Delta_{x}-2 \pi t^{-1} \partial / \partial t$, we have $L u=0$ and $L|u|^{2}=2\left|\nabla_{x} u\right|^{2}=2 \sum_{j=1}^{n}\left|\partial u / \partial x_{j}\right|^{2}$. Making use of notation in [2], we have $S_{\alpha}|u|^{2}=2 \omega_{n} \alpha^{n} \sum_{j=1}^{n} S_{\alpha}^{2}\left(x, K_{j}\right)$, where $K_{j}(x, t)=t \partial u(x, t) / \partial x_{j}(j=1, \cdots, n)$ and $\omega_{n}$ is the measure of the unit sphere in $\boldsymbol{R}^{n}$.

EXAMPLE 3. If $v$ is subharmonic and not identically $-\infty$ in $\boldsymbol{R}_{+}^{n+1}$, and if $L=\Delta$, then $S_{\alpha} v$ is identical with that in McConnell [7].

We define the non-tangential maximal functions $N_{\alpha} v$ by

$$
\left(N_{\alpha} v\right)(x)=\operatorname{ess} \sup \left|v \chi_{\Gamma_{\alpha}(x)}\right|=\left\|v \chi_{\Gamma_{\alpha}(x)}\right\|_{\infty},
$$


where $\chi_{\Gamma_{\alpha}(x)}$ is the characteristic function of $\Gamma_{\alpha}(x)$ and ess sup is the essential supremum with respect to the Lebesgue measure in $\boldsymbol{R}_{+}^{n+1}$.

For technical reasons, we introduce

$$
\left[S_{\alpha}(W) v\right](x)=\int_{W \cap \Gamma_{\alpha}(x)} s^{1-n} d \mu_{L v}(y, s)
$$

for Borel sets $W \subset \boldsymbol{R}_{+}^{n+1}$. For $E \subset \boldsymbol{R}^{n},|E|$ denotes the Lebesgue measure of $E$. We denote by $B_{n}(x, R)$ the open ball in $\boldsymbol{R}^{n}$ of radius $R$ centered at $x$. For $R>0$, we set $T_{R}=B_{n}(0, R) \times(1 / R, R) \subset R_{+}^{n+1}$, where $o$ denotes the origin in $\boldsymbol{R}^{n}$.

Our main purpose is to obtain the following good $\lambda$ inequalities, which lead to the norm inequalities stated in Introduction.

THEOREM. Let $L$ be the operator defined by (1.1) with an $(n+1)-$ dimensional complex square matrix $A$ and complex numbers $b_{1}, \cdots, b_{n+1}$, and let $v$ be a locally integrable function on $\boldsymbol{R}_{+}^{n+1}$ such that $L v$ is a positive Borel measure on $\boldsymbol{R}_{+}^{n+1}$. If $0<\alpha<\beta<\infty$, then

$\left|\left\{x \in \boldsymbol{R}^{n} ;\left[S_{\alpha}\left(T_{R}\right) v\right](x)>\gamma,\left(N_{\beta} v\right)(x) \leqq 1\right\}\right| \leqq c_{1} \exp \left(-c_{2} \gamma\right)\left|\left\{x \in \boldsymbol{R}^{n} ;\left[S_{2 \alpha}\left(T_{R}\right) v\right](x)>1\right\}\right|$ for all $\gamma>1$, where $c_{1}$ and $c_{2}$ are positive constants depending only on $n, \alpha, \beta$ and $L$.

Corollary 1. Let $v$ be the same as in Theorem. For any $0<\alpha$, $\beta<\infty$ and $0<p<\infty$, there exists a constant $C$ depending only on $n$, $\alpha, \beta, L$ and $p$ such that $\left\|S_{\alpha} v\right\|_{p} \leqq C\left\|N_{\beta} v\right\|_{p}$.

COROLLARY 2. Let $v$ be the same as in Theorem and suppose $0<$ $\alpha<\beta<\infty$. Then, for any $0<p<\infty$, there exist positive constants $c$ and $C$ depending only on $n, \alpha, \beta, L$ and $p$ such that

$$
\int_{R^{n}} \exp \left\{c\left(S_{\alpha} v\right)(x) /\left(N_{\beta} v\right)(x)\right\}\left\{\left(S_{\alpha} v\right)(x)\right\}^{p} d x \leqq C\left\|S_{\alpha} v\right\|_{p}^{p} .
$$

When $L=\Delta, v=|u|^{2}$ and $u$ is harmonic, the above Corollary 2 was obtained by Murai-Uchiyama [8], as an improvement of Fefferman-GundySilverstein-Stein [5].

We shall prove Theorem following Uchiyama [9]. In the process, the following lemma plays an important role. It is an extension of Lemma 1 in [9] but is simpler: We do not have to control $\nabla v$ by $N_{\beta} v$ as in [9].

To state the lemma, we let $W_{\alpha}(E)=\bigcup\left\{\Gamma_{\alpha}(x) ; x \in E\right\}$ for $E \subset \boldsymbol{R}^{n}$.

Lemma. Let $v, \alpha$ and $\beta$ be the same as in Theorem, and let $E=$ $\left\{x \in \boldsymbol{R}^{n} ;\left(N_{\beta} v\right)(x) \leqq 1\right\}$. If $\nu$ is defined by

$$
\nu(W)=\int_{W_{\alpha}(E) \cap W} t d \mu_{L v}(x, t)
$$


for Borel sets $W \subset \boldsymbol{R}_{+}^{n+1}$, then $\nu$ is a Carleson measure and the Carleson norm $\|\nu\|_{c}=\sup \left\{\nu\left[I \times\left(0,|I|^{1 / n}\right)\right] /|I| ; I\right.$ is a cube in $\left.\boldsymbol{R}^{n}\right\}$ is bounded by a constant depending only on $n, \alpha, \beta$, and $L$.

2. Proof of Lemma. Let $I$ be a cube in $\boldsymbol{R}^{n}$ and let $l(I)$ be its side length. It is clear that

$$
\nu[I \times(0, l(I))] \leqq \lim _{h \rightarrow+0} \int_{W_{\alpha}(E) \cap W_{\alpha}(I) \cap\left\{R^{n} \times(h, l(I))\right\}} t d \mu_{L v}(x, t) .
$$

For small $h>0$, we define $W$ by

$$
W=W_{(\alpha+\beta) / 2}(E) \cap W_{(\alpha+\beta) / 2}(I) \cap\left\{\boldsymbol{R}^{n} \times([1-\tau] h,[1+\tau] l(I))\right\},
$$

where we take sufficiently small $\tau>0$ so that the following relations hold:

$$
\begin{array}{lll}
B_{n+1}((x, t), \tau t) \subset \Gamma_{(\alpha+\beta) / 2}(o) & \text { for } & (x, t) \in \Gamma_{\alpha}(o), \text { and } \\
B_{n+1}((x, t), \tau t) \cap \Gamma_{(\alpha+\beta) / 2}(o)=\varnothing & \text { for } \quad(x, t) \notin \Gamma_{\beta}(o) .
\end{array}
$$

Let $\rho$ be a non-negative infinitely differentiable function on $\boldsymbol{R}^{n+1}$ such that supp $\rho \subset B_{n+1}(0,1) \subset \boldsymbol{R}^{n+1}$ and its integral over $\boldsymbol{R}^{n+1}$ is equal to 1 . Let $\rho_{\varepsilon}(x, r)=\varepsilon^{-n-1} \rho\left(\varepsilon^{-1} x, \varepsilon^{-1} r\right)$ for $(x, r) \in \boldsymbol{R}^{n+1}$ and $\varepsilon>0$, and let $\chi$ be

$$
\chi(x, t)=\left(\chi_{W} * \rho_{\tau t}\right)(x, t)=\int_{W}(\tau t)^{-n-1} \rho((x-y) / \tau t,(t-s) / \tau t) d y d s
$$

for $(x, t) \in \boldsymbol{R}_{+}^{n+1}$, where $\chi_{W}$ is the characteristic function of $W$. By the property (2.3) and the definition of $W$, we have

$$
\operatorname{supp} \chi \subset\left[I^{*} \times(\{1-\tau\} h /\{1+\tau\},\{1+\tau\} l(I) /\{1-\tau\})\right] \cap\left[W_{\beta}(E) \cap W_{\beta}(I)\right],
$$

where $I^{*}$ is the cube which we obtain by expanding $I\{1+2 \beta(1+\tau) /(1-\tau)\}$ times and has the same center as $I$. We also see that $\chi$ is a non-negative infinitely differentiable function. Furthermore, by (2.2), we have

$$
\chi(x, t)=1 \text { for }(x, t) \in W_{\alpha}(E) \cap W_{\alpha}(I) \cap\left\{\boldsymbol{R}^{n} \times(h, l(I))\right\} .
$$

Therefore, the integral in (2.1) is not greater than

$$
J=\int_{R_{+}^{n+1}} t \chi(x, t) d \mu_{L v}(x, t) .
$$

Since $t \chi(x, t)$ is an infinitely differentiable function with compact support on $\boldsymbol{R}_{+}^{n+1}$, in other words, a test function, we get

$$
J=\int_{W_{0}} L^{*}[t \chi(x, t)] v(x, t) d x d t,
$$

where $W_{0}=\left\{(x, t) ; L^{*}[t \chi(x, t)] \neq 0\right\}$ and

$$
L^{*}=\sum_{j, k=1}^{n+1} a_{j k} \frac{\partial^{2}}{\partial x_{j} \partial x_{k}}-\sum_{j=1}^{n+1} b_{j} \frac{\partial}{\partial x_{j}} \frac{1}{t} .
$$


Elementary calculus shows that

$$
L^{*}[t \chi(x, t)]=t \sum_{j, k=1}^{n+1} a_{j k} \frac{\partial^{2} \chi}{\partial x_{j} \partial x_{k}}(x, t)+\sum_{j=1}^{n+1} c_{j} \frac{\partial \chi}{\partial x_{j}}(x, t),
$$

where $c_{j}=a_{j, n+1}+a_{n+1, j}-b_{j}(j=1, \cdots, n+1)$. Hence, by $(2.4)$ and (2.5), the domain $W_{0}$ of integration is narrow. We can easily check that $\left|\partial^{2} \chi(x, t) / \partial x_{j} \partial x_{k}\right| \leqq C / t^{2}$ and $\left|\partial \chi(x, t) / \partial x_{j}\right| \leqq C / t$ for every $(x, t) \in \boldsymbol{R}_{+}^{n+1}$ and all $j, k=1, \cdots, n+1$ with some constant $C$ depending only on $n, \tau$ and $\rho$. For $(x, t) \in W_{0} \subset \operatorname{supp} \chi$, there exists a point $z \in E$ such that $(x, t) \in \Gamma_{\beta}(z)$ by (2.4). This implies ess $\sup \left|v \chi_{W_{0}}\right|=\left\|v \chi_{W_{0}}\right\|_{\infty} \leqq 1$, where $\chi_{W_{0}}$ is the characteristic function of $W_{0}$. Therefore,

$$
J \leqq C \int_{W_{0}} t^{-1} d x d t
$$

with $C$ depending only on $n, \tau, \rho$ and $L$, but independent of $h$. If we write $W_{0}(x)=\left\{t ;(x, t) \in W_{0}\right\}$ for $x \in I^{*}$, the last integral is equal to

$$
\int_{T^{*}} d x \int_{W_{0}(x)} t^{-1} d t
$$

by Fubini's theorem. We divide $I^{*}$ into three parts $I_{1}^{*}, I_{2}^{*}$ and $I_{3}^{*}$;

$$
\begin{aligned}
& I_{1}^{*}=\left\{x ;(x, h) \in W_{\alpha}(E) \cap W_{\alpha}(I)\right\}, \\
& I_{2}^{*}=\left\{x ;(x, l(I)) \in W_{\alpha}(E) \cap W_{\alpha}(I)\right\}-I_{1}^{*} \text { and } \\
& I_{3}^{*}=I^{*}-\left(I_{1}^{*} \cup I_{2}^{*}\right) .
\end{aligned}
$$

The following inclusion relations are easily checked:

$$
\begin{aligned}
& W_{0}(x) \subset[\{(1-\tau) /(1+\tau)\} h, h] \cup[l(I),\{(1+\tau) /(1-\tau)\} l(I)] \text { for } x \in I_{1}^{*}, \\
& W_{0}(x) \subset[d(x) / \beta, d(x) / \alpha] \cup[l(I),\{(1+\tau) /(1-\tau)\} l(I)] \text { for } x \in I_{2}^{*}, \text { and } \\
& W_{0}(x) \subset[\{\alpha / \beta\} l(I),\{(1+\tau) /(1-\tau)\} l(I)] \text { for } x \in I_{3}^{*} .
\end{aligned}
$$

The function $d$ appearing in the second relation is defined by $d(x)=$ $\max \{$ the distance of $x$ from $E$, the distance of $x$ from $I\}$. By these relations, we have $\int_{W_{0}(x)} t^{-1} d t \leqq C^{\prime}$ for a constant $C^{\prime}$. Therefore,

$$
J \leqq C C^{\prime}\left|I^{*}\right| \leqq C^{\prime \prime}|I|
$$

with a constant $C^{\prime \prime}$ depending only on $n, \alpha, \beta, \tau, \rho$ and $L$, but independent of $h$. This and (2.1) imply $\nu[I \times(0, l(I))] \leqq C^{\prime \prime}|I|$.

3. Proof of Theorem. We use the following known theorems.

THEOREM A (Murai-Uchiyama [8]). If $f \in B M O\left(\boldsymbol{R}^{n}\right)$ and if $\|f\|_{B M O} \leqq 1$, then there exist positive constants $c_{1}^{\prime}$ and $c_{2}^{\prime}$ depending only on $n$ such that 


$$
|\{x ;|f(x)|>\gamma\}| \leqq c_{1}^{\prime} \exp \left(-c_{2}^{\prime} \gamma\right)|\{x ;|f(x)|>1\}| \quad(\gamma>1) .
$$

THEOREM B. Let $\nu$ be a finite Carleson measure on $\boldsymbol{R}_{+}^{n+1}$ with the Carleson norm $\|\nu\|_{c}$ and $K$ be an integrable continuous function on $\boldsymbol{R}^{n}$ satisfying

$$
|K(x+y)-K(x)| \leqq B|y|(1+|x|)^{-n-1} \quad(|x| \geqq 2|y|)
$$

for a constant $B$. Then

$$
\left(S_{K} \nu\right)(x)=\int_{R_{+}^{n+1}} K_{t}(x-y) d \nu(y, t)
$$

exists for almost all $x \in \boldsymbol{R}^{n}$ and $\left\|S_{K} \nu\right\|_{B M O} \leqq C_{n}\left(\|K\|_{1}+B\right)\|\nu\|_{C}$, where $C_{n}$ is a constant depending only on $n$, and $K_{t}(x)=t^{-n} K\left(t^{-1} x\right)$ for $(x, t) \in \boldsymbol{R}_{+}^{n+1}$.

When $K_{t}(x)$ is the Poisson kernel on $\boldsymbol{R}_{+}^{n+1}, S_{K} \nu$ is the balayage of $\nu$. In this case, Theorem $B$ is proved in [6, pp. 229-230]. The proof can be applied to our case.

Now, we begin the proof of our Theorem. Let $\psi$ be an infinitely differentiable function on $\boldsymbol{R}^{n}$ satisfying $0 \leqq \psi \leqq 1, \psi(x)=1(|x| \leqq 1)$ and $\psi(x)=0 \quad(|x| \geqq 2)$. Using this $\psi$, we set $K(x)=\psi(x / \alpha)$ and $K_{t}(x)=$ $t^{-n} K\left(t^{-1} x\right)$ for $x \in \boldsymbol{R}^{n}$ and $t>0$. Then

$$
t^{n} K_{t}(x)=1 \text { for }(x, t) \in \Gamma_{\alpha}(0) \text {, and } t^{n} K_{t}(x)=0 \text { for }(x, t) \notin \Gamma_{2 \alpha}(0) \text {. }
$$

We set $E=\left\{x ;\left(N_{\beta} v\right)(x) \leqq 1\right\}$ and define the measure $\nu$ exactly as in Lemma. If we write $\nu_{R}(W)=\nu\left(W \cap T_{R}\right)$ for $W \subset \boldsymbol{R}_{+}^{n+1}$, then, by the above relations, we get

$$
\left[S_{\alpha}\left(W_{\alpha}(E) \cap T_{R}\right) v\right](x) \leqq\left(S_{K} \nu_{R}\right)(x) \leqq\left[S_{2 \alpha}\left(T_{R}\right) v\right](x)
$$

for all $x \in \boldsymbol{R}^{n}$. Lemma shows that $\nu_{R}$ is a finite Carleson measure and there exists a constant $C_{1}$ depending only on $n, \alpha, \beta$, and $L$ such that $\left\|\nu_{R}\right\|_{C} \leqq\|\nu\|_{C} \leqq C_{1}$. Applying Theorem B to $S_{K} \nu_{R}$, we have $\left\|S_{K} \nu_{R}\right\|_{B M O} \leqq$ $C_{2}\left\|\nu_{R}\right\|_{C}$ with some constant $C_{2}$ depending only on $n$ and $K$. Therefore, Theorem A implies

$$
\left|\left\{x ;\left(S_{K} \nu_{R}\right)(x)>\gamma\right\}\right| \leqq c_{1} \exp \left(-c_{2} \gamma\right)\left|\left\{x ;\left(S_{K} \nu_{R}\right)(x)>1\right\}\right|
$$

for all $\gamma>1$, where $c_{1}$ and $c_{2}$ are positive constants depending only on $n, C_{1}$ and $C_{2}$. Since $\left[S_{\alpha}\left(T_{R}\right) v\right](x)=\left[S_{\alpha}\left(W_{\alpha}(E) \cap T_{R}\right) v\right](x)$ for $x \in E$, Theorem follows from (3.1) and (3.2).

3. Proofs of Corollaries 1 and 2. To prove Corollary 1, we may assume $0<\alpha<\beta<\infty$ without loss of generality (cf. Fefferman-Stein [4, Lemma 1]). It is easy to derive

$$
\left\|S_{\alpha}\left(T_{R}\right) v\right\|_{p}^{p} \leqq \gamma^{p}\left\{\left\|N_{\beta} v\right\|_{p}^{p}+c_{1} \exp \left(-c_{2} \gamma\right)\left\|S_{2 \alpha}\left(T_{R}\right) v\right\|_{p}^{p}\right\}
$$


for any $\gamma>1$ from Theorem with the same constants $c_{1}$ and $c_{2}$ as those in it (cf. Uchiyama [9]). For the second term in the braces, we have $\left\|S_{2 \alpha}\left(T_{R}\right) v\right\|_{p}^{p} \leqq C_{0}\left\|S_{\alpha}\left(T_{R}\right) v\right\|_{p}^{p}$ for a constant $C_{0}$ depending only on $n, \alpha$ and $p$ as in the proof of Proposition 4 in [3]. Our assumption on $v$ and the fact that the support of $\left[S_{\alpha}\left(T_{R}\right) v\right]$ is compact give $\left\|S_{\alpha}\left(T_{R}\right) v_{p}\right\|<\infty$. Therefore, we have

$$
\left\{1-C_{0} c_{1} \gamma^{p} \exp \left(-c_{2} \gamma\right)\right\}\left\|S_{\alpha}\left(T_{R}\right) v\right\|_{p}^{p} \leqq \gamma^{p}\left\|N_{\beta} v\right\|_{p}^{p} .
$$

Taking a suitable $\gamma$ and letting $R$ tend to infinity, we have the conclusion of Corollary 1.

Corollary 2 is an analogue of a result of Murai-Uchiyama [8]. We give here a proof slightly different from theirs.

Let $c_{2}$ be the same constant as that in Theorem. As the constant $c$ in Corollary 2, we may take any number $c$ such that $0<c<c_{2}$. Take an infinitely differentiable function $\chi$ on $(0, \infty)$ such that $\chi(t)=0$ for $0<t<1$, and $\chi(t)=1$ for $t>2$, and set $\phi_{0}(t)=\chi(t) \exp (c t)$ and $\phi_{1}(t)=$ $\{1-\chi(t)\} \exp (c t)$ for $0<t<\infty$. Then

$$
\begin{aligned}
& \int_{R^{n}} \exp \left\{c\left(S_{\alpha} v\right)(x) /\left(N_{\beta} v\right)(x)\right\}\left\{\left(S_{\alpha} v\right)(x)\right\}^{p} d x \\
& \quad \leqq \int_{R^{n}} \Phi\left[\left(S_{\alpha} v\right)(x),\left(N_{\beta} v\right)(x)\right] d x+\int_{R^{n}} \phi_{1}\left[\left(S_{\alpha} v\right)(x) /\left(N_{\beta} v\right)(x)\right]\left\{\left(S_{\alpha} v\right)(x)\right\}^{p} d x,
\end{aligned}
$$

where $\Phi(t, u)=\phi_{0}(t / u) t^{p}$. The second term is bounded by $\exp (2 c)\left\|S_{\alpha} v\right\|_{p}^{p}$. As for the first term, by Fatou's theorem, it is sufficient to estimate

$$
I_{R}=\int \Phi\left(\left[S_{\alpha}\left(T_{R}\right) v\right](x),\left(N_{\beta} v\right)(x)\right) d x .
$$

If $\left(N_{\beta} v\right)(x)=0$, then $\left[S_{\alpha}\left(T_{R}\right) v\right](x)=0$. Therefore, we may regard the integral of $I_{R}$ to be taken over $\left\{x ;\left(N_{\beta} v\right)(x) \neq 0\right\}$. By the definition of $\Phi$, we have

$$
I_{R}=\iint_{0<u<t}-\Phi_{t u}(t, u)\left|\left\{x ;\left[S_{\alpha}\left(T_{R}\right) v\right](x)>t,\left(N_{\beta} v\right)(x) \leqq u\right\}\right| d t d u,
$$

where $\Phi_{t u}=\partial^{2} \Phi / \partial t \partial u$. By Theorem, the right hand side is bounded by

$$
c_{1} \int_{0}^{\infty}\left|\left\{x ;\left[S_{2 \alpha}\left(T_{R}\right) v\right](x)>u\right\}\right| d u \int_{u}^{\infty}\left|\Phi_{t u}(t, u)\right| \exp \left(-c_{2} t / u\right) d t .
$$

Since $\Phi_{t u}(t, u)=-u^{p-1}\left\{(1+p)(t / u)^{p} \phi_{0}^{\prime}(t / u)+(t / u)^{p+1} \phi_{0}^{\prime \prime}(t / u)\right\} / u$, the inner integral is equal to

$$
C_{1}=u^{p-1} \int_{1}^{\infty}\left|(1+p) t^{p} \phi_{0}^{\prime}(t)+t^{p+1} \phi_{0}^{\prime \prime}(t)\right| \exp \left(-c_{2} t\right) d t .
$$

Because of $0<c<c_{2}$, the last integral is finite, and so 


$$
I_{R} \leqq C_{1} c_{1} p^{-1}\left\|S_{2 \alpha}\left(T_{R}\right) v\right\|_{p}^{p} \leqq C_{0} C_{1} c_{1} p^{-1}\left\|S_{\alpha} v\right\|_{p}^{p} .
$$

This completes the proof of Corollary 2.

\section{REFERENCES}

[1] D. L. Burkholder AND R.F. Gundy, Distribution function inequalities for the area integral, Studia Math. 44 (1972), 527-544.

[2] A.P. Calderón and A. TorChinsky, Parabolic maximal functions associated with a distribution, Adv. in Math. 16 (1975), 1-64.

[3] R. R. Colfman, Y. Meyer and E. M. Stein, Some new function spaces and their applications to harmonic analysis, J. Funct. Anal. 62 (1985), 304-335.

[4] C. Feffermman and E. M. Stein, $H^{p}$ spaces of several variables, Acta Math. 129 (1972), 137-193.

[5] R. Fefferman, R. Gundy, M. Silverstein and E. M. Stein, Inequalities for ratios of functionals of harmonic functions, Proc. Nat. Acad. Sci. U.S.A. 79 (1982), 7958-7960.

[6] J. B. Garnett, Bounded Analytic Functions, Pure and Applied Math. 96, Academic Press, New York-London-Toronto-Sydney-San Francisco, 1981.

[7] T. R. McConNelL, Area integrals and subharmonic functions, Indiana Univ. Math. J. 33 (1984), 289-303.

[8] T. Murai and A. Uchiyama, Good $\lambda$ inequalities for the area integral and the nontangential maximal function, Studia Math. 83 (1986), 251-262.

[9] A. Uchiyama, On McConnell's inequality for functionals of subharmonic functions, Pacific J. Math 128 (1987), 367-377.

Department of MATHEMatics

College of General Education

TôHOKU UNIVERSITY

Kawauchi, Sendai, 980

JAPAN 